\title{
Characteristics and potential malignancy of colorectal juvenile polyps in adults: a single-center retrospective study in China
}

\author{
Jie Dong ${ }^{1}$, Tian-Shi Ma ${ }^{2}$, Yuan-Hong $\mathrm{Xu}^{3}$, Peng Li ${ }^{1}$, Wan-Yuan Chen ${ }^{2}$, Jiang-Feng Tu ${ }^{1}$ and You-Wei Chen ${ }^{1 *}$ (])
}

\begin{abstract}
Background: Colorectal juvenile polyps are rare and generally considered benign in adults. Carcinogenesis or neoplastic changes are rarely mentioned in the literature. We systematically evaluated the characteristics and potential malignancy of colorectal juvenile polyps in adults.

Methods: We retrospectively reviewed the medical records of 103 adults diagnosed with colorectal juvenile polyps from September 2007 to May 2020 at our hospital. The characteristics, endoscopic findings, occurrence of intraepithelial neoplasia, carcinogenesis and diagnostic value of chicken skin mucosa (CSM) were analyzed.

Results: The average age of patients with juvenile polyps was 43.2 years (range, 19 to 78 years). A total of 101 patients $(101 / 103,98.1 \%)$ had a single juvenile polyp, and two patients had multiple polyps (107 polyps in total). Polyp sizes ranged from 0.5 to $5 \mathrm{~cm}$. One $(1 / 107,0.9 \%)$ juvenile polyp was cancerous, and 7 (7/107, 6.5\%) developed low-grade intraepithelial neoplasia. Neoplasia and cancerization did not appear in the two patients with multiple polyps. A 27-year-old female had a 2-cm polyp with well-differentiated adenocarcinoma in the mucosa in the sigmoid colon with erosion on the surface. CSM was observed adjacent to 17 polyps, which were all located in the rectum and sigmoid colon, and one polyp showed low-grade intraepithelial neoplasia.
\end{abstract}

Conclusions: Colorectal juvenile polyps occur in a wide range of locations and in variable sizes and numbers. These polyps are solitary in most patients and have neoplastic potential. CSM is not a tumorigenic marker in colorectal juvenile polyps and usually occurs in the distant colorectum. Colorectal juvenile polyps in adults may progress from lowgrade intraepithelial neoplasia to high-grade intraepithelial neoplasia and then to carcinoma and should be treated when discovered and regularly followed as colorectal adenomas.

Keywords: Colorectal juvenile polyps in adult patients, Characteristics, Potential malignancy, Chicken skin mucosa, Treatment

\section{Background}

Juvenile polyps are a type of hamartoma. Although these polyps are the most common type in children [1], colorectal juvenile polyps are rare in adults $[2,3]$. The

\footnotetext{
*Correspondence: cyw@zju.edu.cn

1 Cancer Center, Department of Gastroenterology, Zhejiang Provincial People's Hospital (Affiliated People's Hospital, Hangzhou Medical College), No. 158, Shangtang Road, Hangzhou 310014, Zhejiang Province, China Full list of author information is available at the end of the article
}

occurrence rate of juvenile polyps in children and adolescents is $2 \%$, which accounts for the majority (approximately $80-90 \%)$ of polyps in paediatric patients [1, 3 , 4]. Hyperplastic polyps and adenomas are the two most common types of polyps in adults. Less than $1 \%$ of juvenile polyps occur in adults [5], and there have been few studies on juvenile polyps in adults. Juvenile polyposis syndrome (JPS) is generally characterized by multiple hamartomatous polyps throughout the 
gastrointestinal tract, and it is considered an autosomal dominant disorder. JPS is accompanied by an increased risk of colorectal and gastric cancer [6, 7]. Unlike JPS, sporadic juvenile polyps in the colon are often solitary and rarely undergo malignant transformation $[1,4,8]$. However, sporadic juvenile polyps may also exhibit dysplasia $[5,9,10]$. Previous studies have primarily focused on juvenile polyps in children. However, juvenile polyps in adults are rare and have been less investigated. Except for research from Denmark [5], there have been few studies of adult colorectal juvenile polyps in large populations, especially in the Asia-Pacific area. We performed a retrospective study of adult patients diagnosed with colorectal juvenile polyps in a Chinese population at a single center. Demographic characteristics, clinical symptoms, endoscopic manifestations and pathological results were analyzed. This study summarized the characteristics and evaluated the potential malignancy and carcinogenic factors of colorectal juvenile polyps in adults.

\section{Methods Study design}

A retrospective analysis of the clinical and pathological data of adult patients diagnosed with colorectal juvenile polyps who were admitted to our clinic from September 2007 until May 2020 was performed. The following inclusion criteria were used: (1) age older than 18 years at the time of diagnosis; and (2) pathological diagnosis of colorectal juvenile polyps. The exclusion criteria were juvenile polyposis syndrome (JPS), CronkhiteCanada syndrome (CCS) or other types of polyposis. Patient age at initial diagnosis (years), sex, abdominal pain, diarrhoea, haematochezia, mucus in the stool and other clinical manifestations, the number, maximum diameter $(\mathrm{cm})$, position, polymorphic morphology of polyps defined by the Paris classification [11] [pedunculated type (0-Ip), subpedunculated type (0-Isp) and sessile type (0-Is)], endoscopic features such as mucosal changes near polyps and pathological results were collected and analyzed. Chi-square tests were used to compare detection rates between groups. Spearman rank correlation analyses were used to compare the characteristics of polyps and clinical features. The expression of MutL homologue 1 (MLH1), MutS homologue 2 (MSH2), MutS homologue 6 (MSH6) and postmeiotic segregation increased 2 (PMS2) in the samples was tested using immunohistochemical staining with the EnVision two-step procedure. Ethics approval and consent to participate for the study was obtained from the Ethics Committee of Zhejiang Provincial People's Hospital (IRB No. 2020QT239).

\section{Results}

\section{Subjects}

From 9/2007 to 5/2020, a total of 103 patients with 107 juvenile polyps were included in this study. Patients were divided into two groups according to the number of polyps (1 polyp and $2-4$ polyps). A total of 101 patients (98.1\%) had a single juvenile polyp, and two patients had multiple polyps. Sixty-four patients were male, and 39 were female. The median age was 43.2 years (range 19-78 years). None of the patients had a family history of polyposis. Three patients had a history of cancer. Two of these patients had colon cancer, and one patient had appendix cancer long before they were diagnosed with colorectal juvenile polyps. A history of cancer had no clinical relevance to the incidence of juvenile polyps. One hundred patients were treated with endoscopy. A total of $35.8 \%(19 / 53)$ of patients had positive faecal occult blood test results (Table 1 ).

\section{Clinical manifestations}

Among the 103 patients, 18 (16.8\%) patients complained of abdominal pain, 11 (10.3\%) patients visited doctors for diarrhoea, 45 (42.1\%) patients experienced bloody stool, and $5(4.7 \%)$ patients had mucus in their stool. Thirty-five (32.7\%) patients had no complaints. Juvenile polyps were found on colonoscopy examination (Table 1).

\section{Endoscopic features}

A total of 101 patients (98.1\%) had a solitary juvenile polyp, and two patients had three polyps. Of the 107 polyps found, most were located in the sigmoid (38, $35.5 \%)$ and rectum $(35,32.7 \%)$, while $1(0.9 \%)$ polyp was in the ileocecum, $10(9.3 \%)$ polyps were in the ascending colon, 10 (9.3\%) polyps were in the transverse colon, and $13(12.1 \%)$ polyps were in the descending colon. The size of the polyps ranged from 0.5 to $5.0 \mathrm{~cm}$. The majority $(43 ; 40.2 \%)$ of polyps were $1-1.9 \mathrm{~cm}$, but $34(31.8 \%)$ polyps were less than $1 \mathrm{~cm}, 19$ (17.8\%) polyps were from 2.0 to $2.9 \mathrm{~cm}$, and $11(10.3 \%)$ polyps were larger than $3 \mathrm{~cm}$. Eighteen (16.8\%) polyps were Paris 0-Is polyps, 39 (36.4\%) polyps were Paris 0-Isp polyps, and the other 50 (46.7\%) were Paris 0-Ip, which accounted for the majority of polyps. Fifty-four (50.5\%) polyps showed a reddish surface. Chicken skin mucosa (CSM) was observed adjacent to 17 polyps, which were all located in the rectum and sigmoid colon and accounted for $23.2 \%$ (17/73) of all rectosigmoid juvenile polyps (Table 1). Patient age, sex, abdominal pain, and diarrhoea were not associated with polyp location, size or polymorphic morphology. Haematochezia was positively correlated with polyp size $(P<0.001)$ and polymorphic morphology $(P=0.005)$. Mucus in the stool was positively correlated with polyp size $(P=0.001)$. 0 -Ip polyps were more likely to be 
Table 1 Summary of patients' conditions and medical procedures

\begin{tabular}{|c|c|}
\hline \multicolumn{2}{|l|}{ Characteristic } \\
\hline \multicolumn{2}{|l|}{ Age, years } \\
\hline Mean & 43.2 \\
\hline Range & $19-78$ \\
\hline \multicolumn{2}{|l|}{ Sex, n } \\
\hline Male & 64 \\
\hline Female & 39 \\
\hline \multicolumn{2}{|l|}{ Clinical manifestations, $\mathrm{n}$} \\
\hline Abdominal pain & 18 \\
\hline Diarrhoea & 11 \\
\hline Bloody stool & 45 \\
\hline Mucous stool & 5 \\
\hline \multicolumn{2}{|l|}{ Numbers of polyps, $n$} \\
\hline Single & 101 \\
\hline Multiple & 2 \\
\hline \multicolumn{2}{|l|}{ Location, n } \\
\hline Ileocecum & 1 \\
\hline Ascending colon & 10 \\
\hline Transverse colon & 10 \\
\hline Descending colon & 13 \\
\hline Sigmoid & 38 \\
\hline Rectum & 35 \\
\hline \multicolumn{2}{|l|}{ Gross appearance, $\mathrm{n}$} \\
\hline Paris 0-Is & 18 \\
\hline Paris 0-Isp & 39 \\
\hline Paris 0-Ip & 50 \\
\hline \multicolumn{2}{|l|}{ Maximum diameter $(\mathrm{cm}), \mathrm{n}$} \\
\hline $0-0.9$ & 34 \\
\hline $1-1.9$ & 43 \\
\hline $2-2.9$ & 19 \\
\hline$\geq 3$ & 11 \\
\hline \multicolumn{2}{|l|}{ Chicken skin mucosa, $\mathrm{n}$} \\
\hline With & 17 \\
\hline Without & 90 \\
\hline \multicolumn{2}{|l|}{ Pathological results, $n$} \\
\hline With low-grade intraepithelial neoplasia & 7 \\
\hline With cancerization & 1 \\
\hline \multicolumn{2}{|l|}{ Therapy, n } \\
\hline Endoscopic therapy & 100 \\
\hline Surgery & 3 \\
\hline
\end{tabular}

associated with haematochezia than 0-Isp and 0-Is polyps (Table 2).

\section{Treatment}

One hundred patients underwent endoscopic therapy, including thermal biopsies, loop snare techniques or endoscopic mucosal resection (EMR), and three patients underwent surgery. One patient was diagnosed with
Table 2 Spearman rank correlation analyses of characteristics of polyps and clinical features

\begin{tabular}{llll}
\hline & Location & Size & Paris classification \\
\hline Age & $P=0.056$ & $P=0.866$ & $P=0.577$ \\
Sex & $P=0.883$ & $P=0.061$ & $P=0.504$ \\
Abdominal pain & $P=0.240$ & $P=0.674$ & $P=0.617$ \\
Diarrhoea & $P=0.647$ & $P=0.431$ & $P=0.992$ \\
Haematochezia & $P=0.828$ & $P<0.001$ & $P=0.005$ \\
Mucus in the stool & $P=0.436$ & $P=0.001$ & $P=0.774$ \\
\hline
\end{tabular}

colon cancer before surgery based on the morphology of the mass on endoscopy, but the postoperative specimen was identified as a juvenile polyp with high levels of stromal oedema and focal tubular adenoma with lowgrade intraepithelial neoplasia. The polyp was located in the descending colon and had a maximum diameter of $5.0 \mathrm{~cm}$. One patient complaining of abdominal pain and diarrhoea was diagnosed with colon cancer before surgery and underwent radical surgery. The third patient had three large polyps approximately $5.0 \mathrm{~cm}$ in diameter that could not be treated under endoscopy (Table 1).

\section{Pathological results}

One colorectal juvenile polyp showed focal carcinogenesis, and seven polyps showed low-grade intraepithelial neoplasia. These polyps were all single polyps. Multiple juvenile polyps were not accompanied by cancerization. There were two tubular adenomas with low-grade intraepithelial neoplasia near two juvenile polyps. One adenoma was accompanied by colon cancer. The patient with focal carcinogenesis was a 27-year-old female. Her polyp, which was approximately $2.0 \mathrm{~cm}$ in size, was located in the sigmoid colon and showed erosion on the surface. Immunohistochemical staining of the polyp showed a Ki-67 index of approximately $80 \%$, mutated p53, with diffuse and strongly positive expression, and positive expression of MLH1, MSH2, MSH6 and PMS2, indicating microsatellite stability (Figs. 1, 2). The immunohistochemical results of the seven polyps with lowgrade intraepithelial neoplasia showed an average Ki-67 index of approximately $40 \%$ (Fig. 3), wild-type p53 without overexpression, and MLH1, MSH2, MSH6 and PMS2 expression. The Ki-67 index was approximately $20 \%$ (Fig. 4) in other simple juvenile polyps, with wild-type p53 and no overexpression. Among the 17 polyps with CSM, one $(5.9 \%, 1 / 17)$ polyp showed low-grade intraepithelial neoplasia. Six polyps with low-grade intraepithelial neoplasia and one polyp with carcinogenesis were found among the remaining 90 polyps without CSM. 

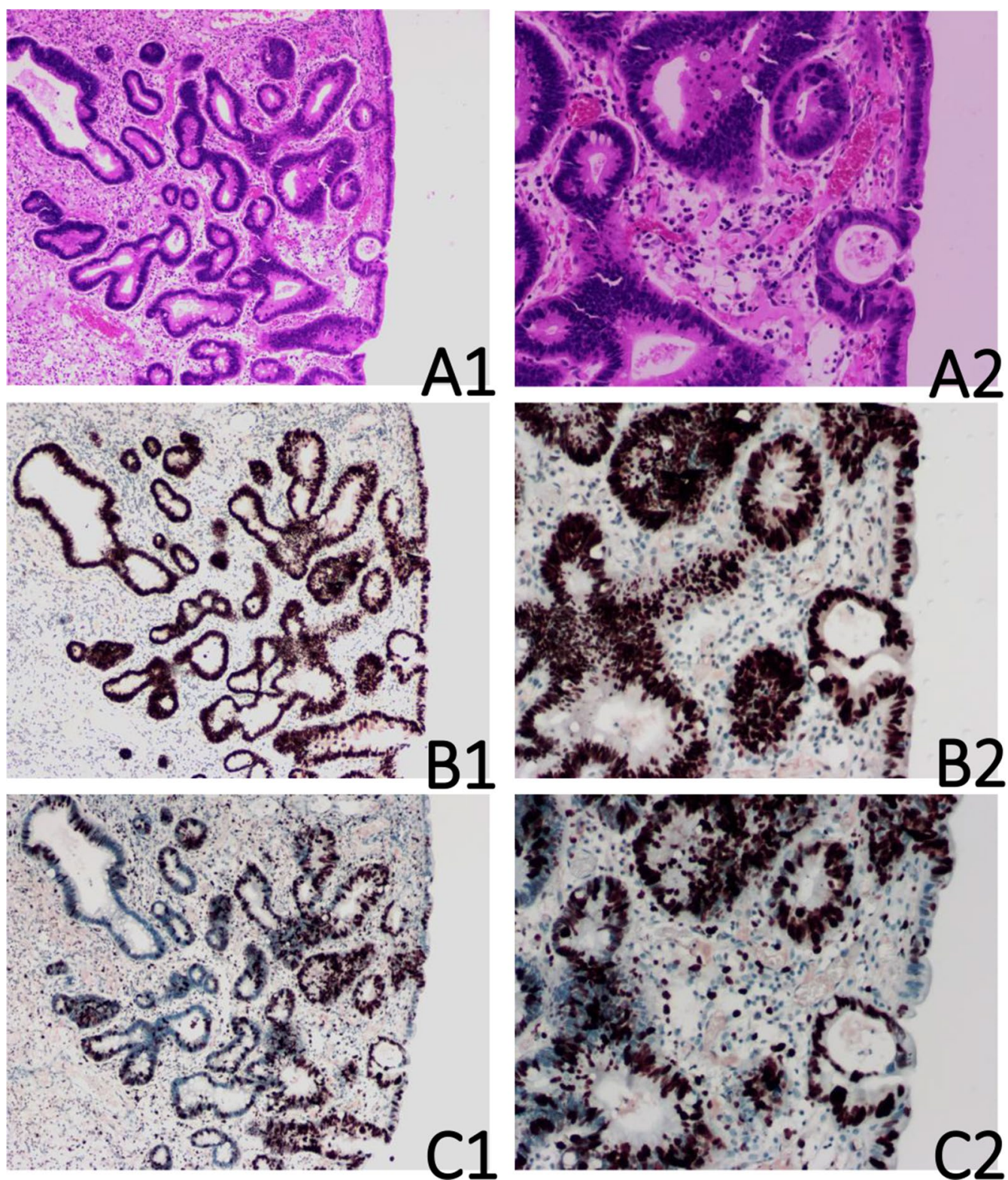

Fig. 1 Juvenile polyp with carcinogenesis. A Haematoxylin and eosin staining (A1 × 40; A2 × 100). B Immunohistochemical staining for p53 showed p53 mutation and overexpression (B1 × 40; B2 × 100). C Immunohistochemical staining for Ki-67 showed an index of 80\% (C1 × 40; $(2 \times 100)$

The chi-square test did not show a significant difference $(\mathrm{P}>0.05)$ (Table 1).

\section{Discussion}

To the best of our knowledge, the current study is the largest single center study on colorectal juvenile polyps in adults in the Asia-Pacific area. Colorectal juvenile polyps are rare in adults. The incidence of juvenile polyps in Danish adults ranges from 1:65,000 to 1:40,000 [5]. Based on our study, the average age of onset in adults was
43.2 years. The ratio of males to females was 1.6:1. These findings are consistent with those of previous reports, indicating an average age between 25.5 and 48.9 years [ 5 , 12] and a male:female ratio of $0.8-1.4: 1$ [8].

The clinical manifestations of juvenile polyps are similar to those of other types of polyps and include abdominal pain, rectal bleeding, prolapse, and diarrhoea. Juvenile polyps can occur in any part of the colon, but most polyps in the current study were located in the rectum and sigmoid colon (68.2\%), which 

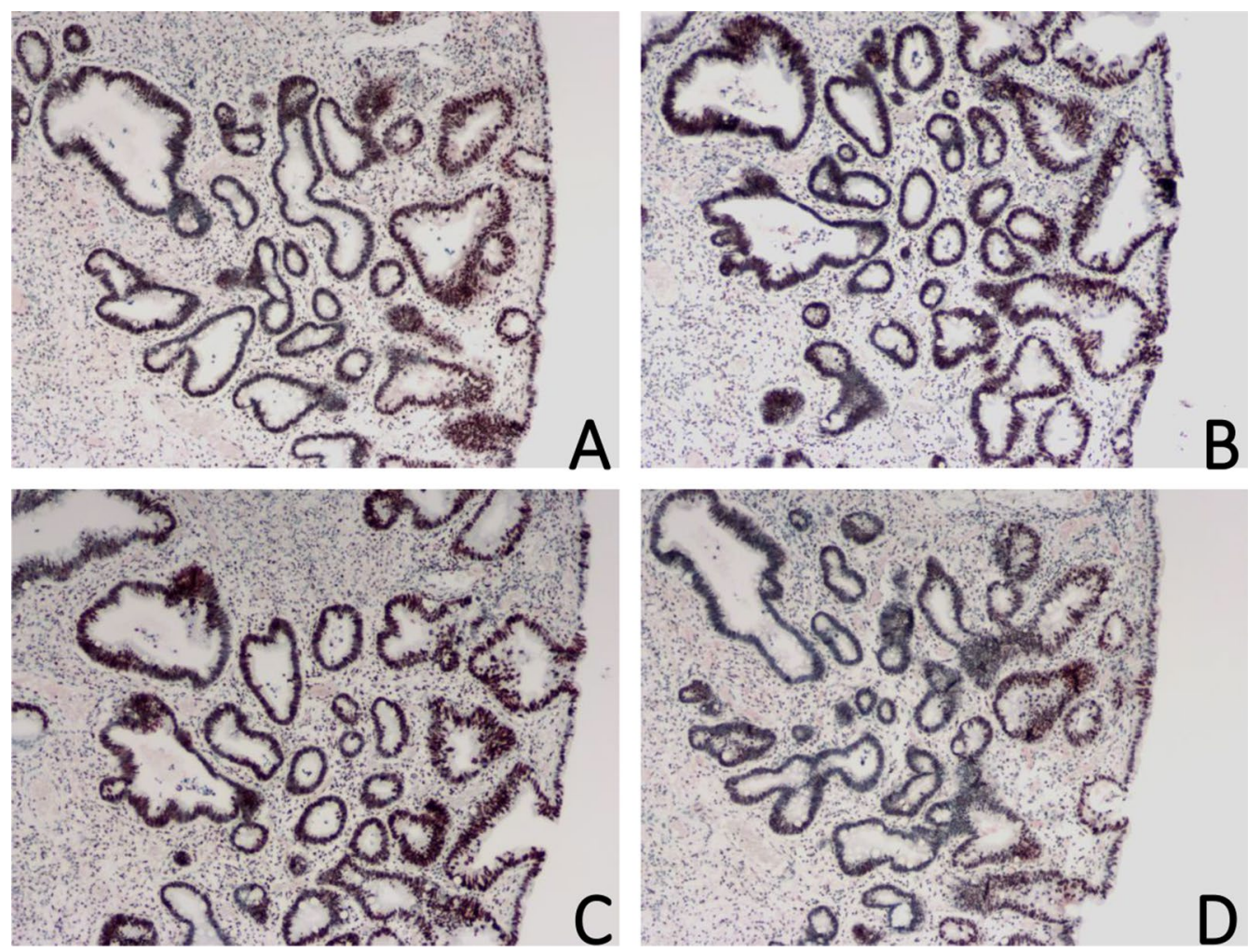

Fig. 2 Juvenile polyp with carcinogenesis. Immunohistochemical staining was positive for A MLH1 ( $\times$ 40), B MSH2 ( $\times$ 40), C MSH6 ( $\times 40)$, and D PMS2 $(\times 40)$
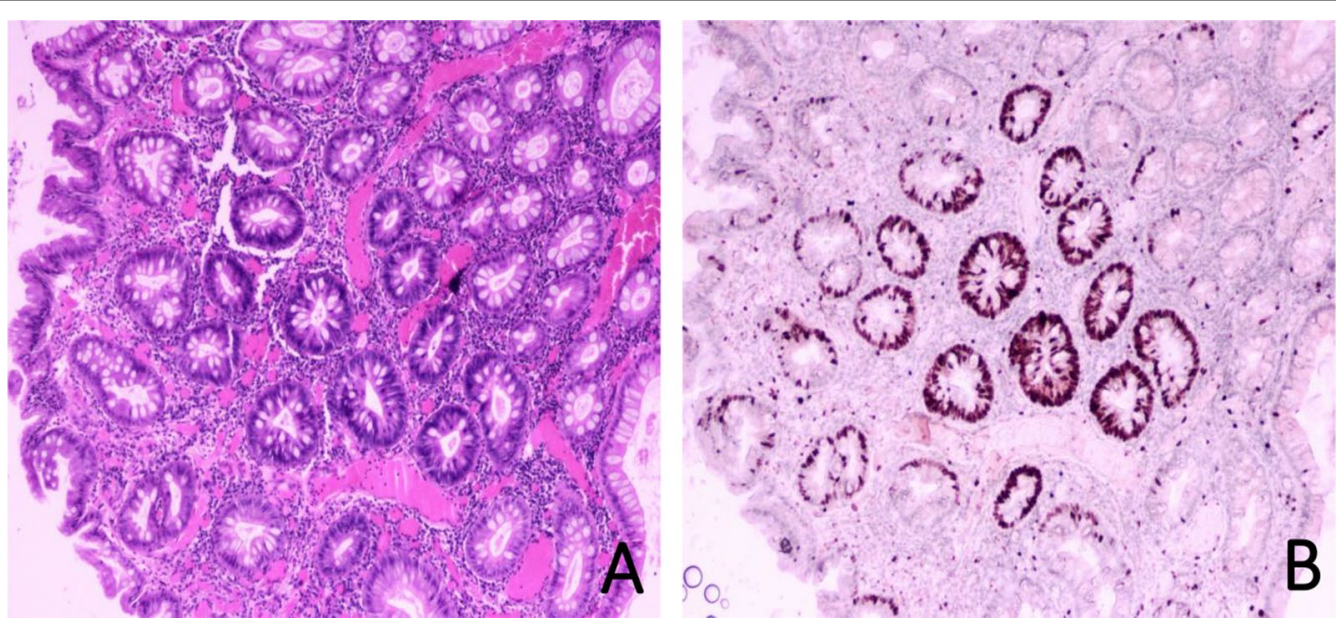

Fig. 3 Juvenile polyp with low-grade intraepithelial neoplasia. A Haematoxylin and eosin staining $(\times 40)$. B Immunohistochemical staining for Ki-67 showed an index of $40 \%(\times 40)$

is similar to the distribution of juvenile polyps reported in children [13] and in a previous study in adults [5]. A total of $98.1 \%(101 / 103)$ of the patients had a single juvenile polyp, which is similar to the rate of $94.9 \%$ reported previously [5]. A total of $42-75.9 \%$ of children had a single polyp [13, 14]. Additionally, $83.2 \%$ of the polyps were Paris 0 -Isp/Ip according to the Paris endoscopic classification $[15,16]$ in our study, which is similar to the finding of a previous report [17]. 

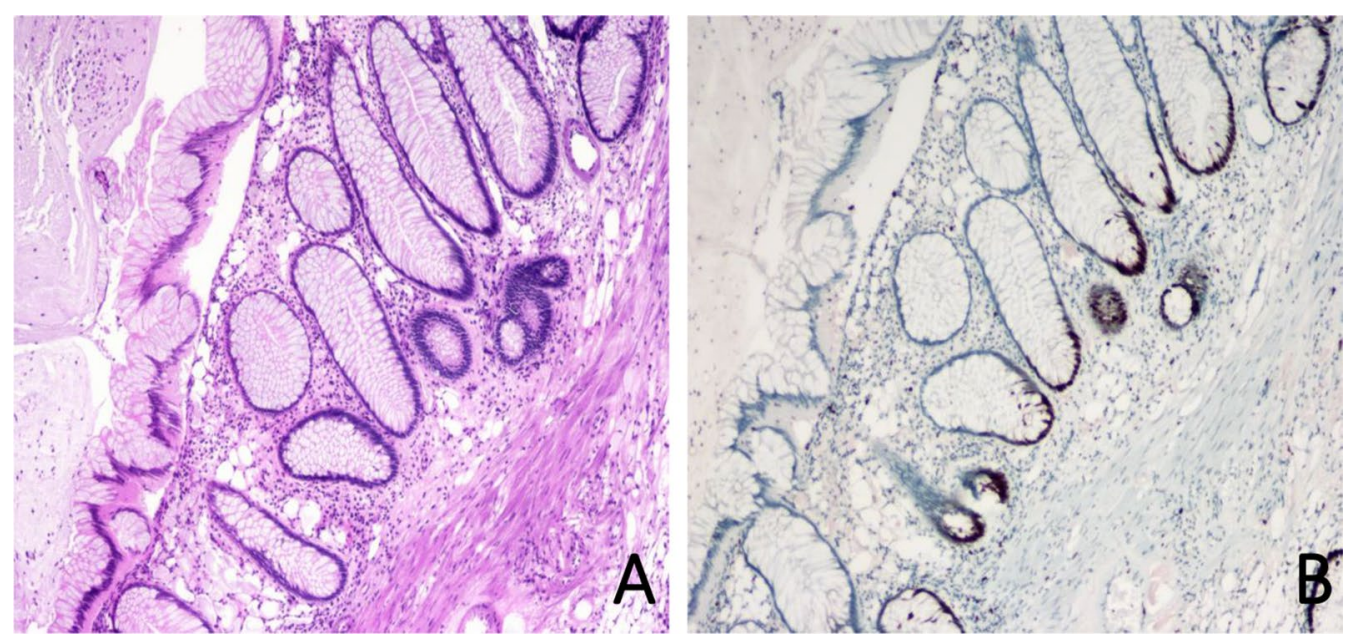

Fig. 4 Simple juvenile polyp. A Haematoxylin and eosin staining ( $\times$ 40). B Immunohistochemical staining for Ki-67 showed an index of 20\% ( $\times$ 40)

The size of juvenile polyps ranged from sessile nodules of a few millimetres to pedunculated lesions up to several centimetres, as determined by endoscopy. Large polyps may be multilobulated, but small polyps are generally round and smooth. Erosion and granulation tissue hyperplasia are often observed on the surface of polyps [18]. Haematochezia and mucus in the stool were correlated with a larger juvenile polyp size, and 0-Ip polyps were more likely to be associated with haematochezia than 0 -Isp and 0-Is polyps by the Paris classification. Small yellow particles were also observed around some juvenile polyps; this manifestation is referred to as CSM [19].

Histopathology is the gold standard for the diagnosis of juvenile polyps because the clinical symptoms and endoscopic features are not entirely typical. This condition primarily manifests as mucinous gland hyperplasia and mucous cysts of different sizes in fibrous tissues. Juvenile polyps are composed of differentiated glandular ducts, and the glandular cavity is dilated to varying degrees. This dilation is generally accompanied by interstitial hyperplasia and the infiltration of large numbers of inflammatory cells, such as lymphocytes, plasma cells, neutrophils and eosinophils, in the stroma. These characteristics distinguish juvenile polyps from juvenile polyposis and Peutz-Jeghers syndrome.

Juvenile polyps are a type of hamartoma with minimal risk $[8,20,21]$. However, the potential of solitary or sporadic juvenile polyps to develop into cancer is not clear. Only a few cases of carcinogenesis from solitary or sporadic juvenile polyps have been described in the literature. Intramucosal carcinoma arising within a solitary juvenile polyp is regarded as 'a wolf in sheep's clothing' $[17,22]$. Other researchers $[10,23,24]$ reported three cases of signet ring cell carcinoma in juvenile polyps.
One $(0.9 \%)$ juvenile polyp with focal carcinogenesis and seven $(6.5 \%)$ polyps with low-grade intraepithelial neoplasia were identified in our study. These polyps were all single polyps. Neoplasia or cancerization did not appear in the two patients with multiple polyps, which is consistent with the finding in a previous report in children [14] that increased numbers of polyps at presentation did not predict further polyp development.

The incidence of adenomatous changes in juvenile polyps is not clear. The polyp with focal carcinogenesis showed higher Ki-67 and p53 expression levels than the seven polyps with low-grade intraepithelial neoplasia. These seven polyps showed higher Ki-67 expression than simple polyps. As previously reported, the expression of p53 and Ki-67 may be used as prognostic factors for adenomas, with high cell proliferation suggesting more aggressive behaviour. Higher levels of p53 and Ki-67 expression are found in adenomas with high-grade dysplasia $[25,26]$.

Based on the results of the immunohistochemical markers mentioned above, we hypothesized that juvenile polyps progress from low-grade intraepithelial neoplasia to high-grade intraepithelial neoplasia and then to carcinoma. A study of 213 paediatric patients found adenomatous changes that were suggestive of the same progression [14]. Based on these findings, the risk of carcinogenesis and the route of cancerization are independent of age and the number of polyps. Based on previous studies and our research, sporadic juvenile polyps might carry an inherent potential for malignancy.

CSM was first identified in 1998 [27] and was described as specific mucosal morphological changes adjacent to colorectal neoplasms. CSM is characterized by a speckled pattern of pale-yellow colorectal mucosa on 
endoscopy. The prevalence of CSM was reported to be $30.7 \%(225 / 733)$ in patients with adenomas. Adenomas with CSM exhibited more high-grade dysplasia and carcinoma than adenomas without CSM, higher expression of Ki-67, COX2 protein and survivin, and lower expression of caspase-3, which indicated the carcinogenetic progression of colorectal adenomas [28, 29]. Therefore, CSM is generally considered a tumour marker in colorectal adenomas. In contrast, since the level of Ki- 67 or p53 expression are not increased in juvenile polyps with CSM in children, CSM is not regarded a marker for subsequent malignancy [30,31]. In our study, there was no difference in the incidence of neoplasia or tumorigenesis between polyps with or without CSM. Hence, CSM was not identified as a tumorigenic marker of colorectal juvenile polyps as it has been in children.

Endoscopic polypectomy is the main treatment for colorectal juvenile polyps. Thermal biopsies, loop snare techniques, EMR, and endoscopic submucosal dissection (ESD) are safe and effective for sporadic, semipedunculated or sessile large juvenile polyps. However, colectomy may be beneficial for multiple or diffuse juvenile polyps, very large polyps, or polyps suspected of malignant transformation [32,33]. Even though juvenile polyps in adults are rare, they should be treated when discovered, with regular follow-up as for colorectal adenomas, as improved compliance with follow-up reduces the risk of carcinogenesis.

\section{Conclusion}

This study is the largest single-center study of the characteristics and potential malignancy of colorectal juvenile polyps in adults in the Asia-Pacific area. Even though colorectal juvenile polyps are often considered benign, they still carry a risk of malignancy. We found a $0.9 \%$ $(1 / 107)$ incidence of cancer and a $6.5 \%$ (7/107) incidence of low-grade intraepithelial neoplasia in colorectal juvenile polyps. Unlike in cases of CSM-related adenoma, CSM was not a tumorigenic marker in cases of colorectal juvenile polyps. Juvenile polyps may progress from lowgrade intraepithelial neoplasia to high-grade intraepithelial neoplasia and then to carcinoma and should be treated cautiously when discovered, with regular followup as for colorectal adenomas.

\footnotetext{
Abbreviations

CSM: Chicken skin mucosa; JPS: Juvenile polyposis syndrome; CCS: Cronkhite-Canada syndrome; MLH1: MutL homologue 1; MSH2: MutS homologue 2; MSH6: MutS homologue 6; PMS2: Postmeiotic segregation increased 2; EMR: Endoscopic mucosal resection; COX-2: Cyclooxygenase-2; ESD: Endoscopic submucosal dissection.
}

Acknowledgements

We are grateful to Yi Chen for chart review.

\section{Authors' contributions}

JD reviewed the literature, designed the study, and drafted the paper. YHX performed the literature review and was responsible for collecting the medical data. PL and JFT reviewed the literature and analyzed the data. TSM and WYC provided the pathological results. YWC designed the study with JD and revised the manuscript. All authors read and approved the final manuscript.

\section{Funding}

This work was supported by the Medical Health Science and Technology Project of Zhejiang Provincial Health Commission (No. 2021436506) and the General Scientific Research Project of Zhejiang Provincial Education Department (No. Y202044280). The funding body played no role in the design of the study, collection, analysis, or interpretation of data, or writing of the manuscript.

\section{Availability of data and materials}

The datasets used and/or analysed during the current study are available from the corresponding author on reasonable request.

\section{Declarations}

\section{Ethics approval and consent to participate}

Ethics approval and consent to participate for the study was obtained from the Ethics Committee of Zhejiang Provincial People's Hospital (IRB No. 2020QT239). Informed consent was waived because of the retrospective nature of the study and our Ethics Committee also approved the informed consent waiver. All the study protocol was in accordance with Declaration of Helsinki.

\section{Consent for publication}

Not applicable.

\section{Competing interests}

The authors declare that they have no conflicts of interest.

\section{Author details}

${ }^{1}$ Cancer Center, Department of Gastroenterology, Zhejiang Provincial People's Hospital (Affiliated People's Hospital, Hangzhou Medical College), No. 158, Shangtang Road, Hangzhou 310014, Zhejiang Province, China. ${ }^{2}$ Cancer Center, Department of Pathology, Zhejiang Provincial People's Hospital (Affiliated People's Hospital, Hangzhou Medical College), No.158, Shangtang Road, Hangzhou 310014, Zhejiang Province, China. ${ }^{3}$ Department of Gastroenterology, The Fifth Affiliated Hospital of Zunyi Medical University, Zhuhai 519000, Guangdong Province, China.

Received: 14 March 2021 Accepted: 11 February 2022

Published online: 21 February 2022

\section{References}

1. Wang S-L, Rana R, Song W-P, He C-Z, Gao H-J, Yang C-Q. Clinicopathological characteristics of children with intestinal polyp in the Southwest of China. Gazzetta Medica Italiana Archivio Per Le Scienze Mediche. 2020;179(1-2):54-8.

2. Horrilleno EG, Eckert C, Ackerman LV. Polyps of the rectum and colon in children. Cancer. 1957;10(6):1210-20.

3. Chow E. MACRAE F: a review of Juvenile Polyposis Syndrome. J Gastroenterol Hepatol. 2005;20(11):1634-40.

4. Zbuk KM, Eng C. Hamartomatous polyposis syndromes. Nat Clin Pract Gastroenterol Hepatol. 2007;4(9):492-502.

5. Jelsig AM, Ousager LB, Brusgaard K, Qvist N. Juvenile polyps in Denmark from 1995 to 2014. Dis Colon Rectum. 2016;59(8):751-7.

6. Brosens LA, van Hattem A, Hylind LM, lacobuzio-Donahue C, Romans KE, Axilbund J, Cruz-Correa M, Tersmette AC, Offerhaus GJ, Giardiello FM. Risk of colorectal cancer in juvenile polyposis. Gut. 2007;56(7):965-7.

7. Latchford AR, Neale K, Phillips RK, Clark SK. Juvenile polyposis syndrome: a study of genotype, phenotype, and long-term outcome. Dis Colon Rectum. 2012;55(10):1038-43. 
8. Nugent KP, Talbot IC, Hodgson SV, Phillips RK. Solitary juvenile polyps: not a marker for subsequent malignancy. Gastroenterology. 1993;105(3):698-700.

9. Dickey W, Alderdice J, McConnell B. Dysplastic change in the solitary juvenile polyp. Endoscopy. 1996;28(7):641.

10. Olafsdottir I, Nemeth A, Lorinc E, Toth E, Agardh D. Value of fecal calprotectin as a biomarker for juvenile polyps in children investigated with colonoscopy. J Pediatr Gastr Nutr. 2016;62(1):43-6.

11. Japanese Society for Cancer of the Colon and Rectum. Japanese classification of colorectal, appendiceal, and anal carcinoma: the $3 \mathrm{~d}$ English Edition [Secondary Publication]. J Anus Rectum Colon. 2019;3(4):175-95.

12. Roth SI, Helwig EB. Juvenile polyps of the colon and rectum. Cancer. 1963:16:468-79.

13. Lee BG, Shin SH, Lee YA, Wi JH, Lee YJ, Park JH. Juvenile polyp and colonoscopic polypectomy in childhood. Pediatric Gastroenterol Hepatol Nutr. 2012;15(4):250-5.

14. Ibrahimi N, Septer SS, Lee BR, Garola R, Shah R, Attard TM. Polyp characteristics of nonsyndromic and potentially syndromic juvenile polyps: a retrospective cohort analysis. J Pediatr Gastroenterol Nutr. 2019;69(6):668-72.

15. Lanza G Jr. ras p21 oncoprotein expression in human colonic neoplasia-an immunohistochemical study with monoclonal antibody RAP-5. Histopathology. 1988;12(6):595-609.

16. Lostal Gracia I, Fleta Zaragozano J, Sarria Chueca A, Dominguez Cunchillos M, Tejedo Grafia V, Ortego Fernandez de Retama J, Soria Navarro J, Suarez Alzamora M, Bueno Sanchez M. Adenomatous degeneration in a prepuberal patient with generalized juvenile polyposis and angiomas of the oral mucosa. An Esp Pediatr. 1988;29(1):61-4.

17. Kang SH, Chung WS, Hyun CL, Moon HS, Lee ES, Kim SH, Sung JK, Lee BS, Jeong HY. A rare case of a signet ring cell carcinoma of the colon mimicking a juvenile polyp. Gut Liver. 2012;6(1):129-31.

18. Brosens LA, Langeveld D, van Hattem WA, Giardiello FM, Offerhaus GJA. Juvenile polyposis syndrome. World J Gastroenterol. 2011;17(44):4839-44.

19. Hirotani A, Sakai E, Nakajima A, Kawana K, Nagase H. Endoscopic findings of atypical juvenile colonic polyps. Gastrointest Endosc. 2016:83(2):476-7.

20. Ye XZ. Immunohistochemical and histochemical study on colorectal adenomas and polyps. Zhonghua Bing Li Xue Za Zhi. 1992;21 (3):149-51.

21. Walpole IR, Cullity G. Juvenile polyposis: a case with early presentation and death attributable to adenocarcinoma of the pancreas. Am J Med Genet. 1989;32(1):1-8.

22. Vanoli A, Lucioni M, Biletta E, Chiaravalli AM, Alvisi C, Luinetti O. A wolf in sheep's clothing: a sporadic juvenile polyp of the colon harboring an intramucosal adenocarcinoma. Int J Surg Pathol. 2015;23(7):571-4.

23. Villar Lucas C, Hernando Martin M, Alvarez-Cuenllas B. Signet ring cell carcinoma in a juvenile polyp. Rev Esp Enferm Dig. 2019;111(12):968.

24. Kim HJ, Kang MK, Lee HS, Kim DS, Lee DH. Signet ring cell carcinoma arising from a solitary juvenile polyp in the colon. J Korean Soc Coloproctol. 2010;26(5):365-7.

25. Nussrat FL, Ali HH, Hussein HG, Al-Ukashi RJ. Immunohistochemical expression of ki-67 and p53 in colorectal adenomas: a clinicopathological study. Oman Med J. 2011;26(4):229-34.

26. Sousa WA, Rodrigues LV, Silva RG Jr, Vieira FL. Immunohistochemical evaluation of p53 and Ki-67 proteins in colorectal adenomas. Arq Gastroenterol. 2012;49(1):35-40.

27. Shatz BA, Weinstock LB, Thyssen EP, Mujeeb I, DeSchryver K. Colonic chicken skin mucosa: an endoscopic and histological abnormality adjacent to colonic neoplasms. Am J Gastroenterol. 1998;93(4):623-7.

28. Chung EJ, Lee JY, Choe J, Chang HS, Kim J, Yang DH, Ye BD, Byeon JS, Kim KJ, Yang SK, et al. Colonic chicken skin mucosa is an independent endoscopic predictor of advanced colorectal adenoma. Intest Res. 2015;13(4):318-25.

29. Guan J, Zhao R, Zhang X, Cheng Y, Guo Y, Wang L, Mi L, Liu F, Ma X, Li B. Chicken skin mucosa surrounding adult colorectal adenomas is a risk factor for carcinogenesis. Am J Clin Oncol. 2012;35(6):527-32.

30. Nowicki MJ, Subramony C, Bishop PR, Parker PH. Colonic chicken skin mucosa: association with juvenile polyps in children. Am J Gastroenterol. 2001;96(3):788-92.

31. Merg A, Howe JR. Genetic conditions associated with intestinal juvenile polyps. Am J Med Genet C Semin Med Genet. 2004;129C(1):44-55.

32. Otake K, Uchida K, Inoue M, Matsushita K, Hashimoto K, Toiyama Y, Tanaka K, Nakatani K, Kawai K, Kusunoki M. A large, solitary, semipedunculated gastric polyp in pediatric juvenile polyposis syndrome. Gastrointest Endosc. 2011;73(6):1313-4.

33. Nowicki MJ, Bishop PR. Successful endoscopic removal of an appendiceal polyp in a child with juvenile polyposis syndrome. Gastrointest Endosc. 2011;74(2):441-3.

\section{Publisher's Note}

Springer Nature remains neutral with regard to jurisdictional claims in published maps and institutional affiliations.
Ready to submit your research? Choose BMC and benefit from:

- fast, convenient online submission

- thorough peer review by experienced researchers in your field

- rapid publication on acceptance

- support for research data, including large and complex data types

- gold Open Access which fosters wider collaboration and increased citations

- maximum visibility for your research: over $100 \mathrm{M}$ website views per year

At BMC, research is always in progress.

Learn more biomedcentral.com/submissions 